\title{
Guaranteed cost inequalities for robust stability and performance analysis
}

\author{
Dennis S. Bernstein ${ }^{1, *, \dagger}$ and Scot L. Osburn ${ }^{2}$ \\ ${ }^{1}$ Department of Aerospace Engineering, The University of Michigan, Ann Arbor MI, 48109-2140, USA \\ ${ }^{2}$ The Aerospace Corporation, P.O. Box 92957-M4/972, El Segundo CA, 90009, USA
}

\begin{abstract}
SUMMARY
In this paper, we formulate robust stability and performance bounds in terms of guaranteed cost inequalities. We derive new guaranteed cost bounds for plants with real structured uncertainty, and we reformulate them as linear matrix inequalities (LMIs). In particular, we obtain a shifted linear bound and a shifted inverse bound, and give LMI forms for a shifted bounded real bound, a shifted Popov bound, a shifted linear bound and a shifted inverse bound. Several examples are used to compare the shifted bounds with their unshifted counterparts and to make comparisons among these new bounds and vertex LMI bounds. Copyright (C) 2002 John Wiley \& Sons, Ltd.
\end{abstract}

\section{INTRODUCTION}

For unstructured time-varying or complex uncertainty, the small gain theorem provides a nonconservative test for robust stability [1-6]. For structured and possibly real uncertainty, however, the small gain theorem is known to be conservative, and structured singular value bounds, which involve multipliers and complex scalings, can be used [7,8]. Linear matrix inequalities (LMIs) are also used to guarantee robust stability [9-15].

Within the context of robust $\mathrm{H}_{2}$ performance, the small gain theorem is equivalent to the bounded real bound [1-3], which plays the role of a guaranteed cost bound [4]. Various guaranteed cost bounds have been developed including quadratic and non-quadratic bounds. Quadratic bounds include the bounded real [1-3], positive real [6,16], and Popov bounds [6,17] (see Table I), while non-quadratic bounds include the absolute value and linear bounds [21-23] (see Table II).

In the present paper we reformulate the bounded real, Popov, inverse, shifted bounded real [18], and shifted Popov [19] bounds as guaranteed cost inequalities. In addition, we present two new guaranteed cost bounds, namely, the shifted linear and shifted inverse bounds, which we also reformulate as linear matrix inequalities.

\footnotetext{
*Correspondence to: Dennis S. Bernstein, Department of Aerospace Engineering, University of Michigan, Ann Arbor MI, 48109-2140, U.S.A.

†E-mail: dsbaero@umich.edu
}

Contract/grant sponsor: Air Force Office of Scientific Research; contract/grant number: F49620-98-1-0037

Published online 30 September 2002

Copyright (C) 2002 John Wiley \& Sons, Ltd.
Received 26 June 2000 Revised 15 November 2001 Accepted 14 December 2001 
Table I. Quadratic guaranteed cost bounds.

\begin{tabular}{ll}
\hline Bound & Reference \\
\hline Bounded real & Anderson et al. [1], Noldus [2], Peterson and Hollot [3] \\
Positive real & Anderson [16], Haddad and Bernstein [6] \\
Popov & Haddad and Bernstein [6,17] \\
Shifted bounded real & Tyan and Bernstein [18] \\
Shifted positive real & Tyan and Bernstein [18] \\
Shifted Popov & Kapila et al. [19] \\
Implicit small gain & Haddad et al. [20] \\
\hline
\end{tabular}

Table II. Non-quadratic guaranteed cost bounds.

\begin{tabular}{ll}
\hline Bound & Reference \\
\hline Absolute value & Chang and Peng [21] \\
Linear & Jain [22], Bernstein [23], Kosmidou and Bertrand [24] \\
Inverse & Bernstein and Haddad [4] \\
Double commutator & Tyan et al. [25] \\
Shifted linear & This paper \\
Shifted inverse & This paper \\
\hline
\end{tabular}

The guaranteed cost bounds that we consider are either parameter independent or parameter dependent. Parameter-independent bounds, such as the bounded real bound, use a common Lyapunov function, whereas parameter-dependent bounds, such as the Popov bound, use a family of Lyapunov functions. For polytopic uncertainty we show that the least conservative common (parameter-independent) guaranteed cost bound can be determined by solving an optimization problem involving a set of linear matrix inequalities. The interesting feature of the guaranteed cost bounds is the fact that they give rise to sets of LMIs whose dimensions are less than the dimensions of the vertex LMIs.

The contents of the paper are as follows. In Section 2 we consider the robust analysis problem in a guaranteed $\mathrm{H}_{2}$ cost inequality framework. In Section 3 we consider the use of vertex LMI's to obtain guaranteed $\mathrm{H}_{2}$ cost bounds. In Sections 4 and 5 we review and analyse the shifted bounded real and shifted Popov bounds, while in Sections 6 and 7 we present the shifted linear bound and shifted inverse bound. Finally, in Section 8 several examples are considered to compare the guaranteed cost and vertex LMI bounds.

Proofs can be found in Appendix A.

\section{ROBUST PERFORMANCE AND GUARANTEED COST BOUNDS}

Let $\mathscr{U} \subset \mathbf{R}^{n \times n}$ denote an uncertainty set and consider the uncertain $p \times m$ transfer function $G_{\Delta A}(s)=E(s I-A-\Delta A)^{-1} D$, where $A \in \mathbf{R}^{n \times n}, \Delta A \in \mathscr{U}, D \in \mathbf{R}^{n \times m}$, and $E \in \mathbf{R}^{p \times n}$. If $A+\Delta A$ is asymptotically stable for all $\Delta A \in \mathscr{U}$, then we define the worst-case $\mathrm{H}_{2}$ performance by

$$
J(\mathscr{U}) \triangleq \sup _{\Delta A \in \mathscr{U}}\left\|G_{\Delta A}\right\|_{2}
$$


It follows from standard results that

$$
J(\mathscr{U})=\sup _{\Delta A \in \mathscr{U}} \operatorname{tr} P_{\Delta A} V,
$$

where $V \triangleq D D^{\mathrm{T}}$ and $P_{\Delta A}$ is the unique, non-negative-definite solution to the Lyapunov equation

$$
(A+\Delta A)^{\mathrm{T}} P_{\Delta A}+P_{\Delta A}(A+\Delta A)+R=0,
$$

where $R \triangleq E^{\mathrm{T}} E$.

The following definition will be used to construct bounds for $J(\mathscr{U})$.

\section{Definition 1}

Let $\mathscr{N} \subseteq \mathbf{S}^{n}, \Omega: \mathscr{N} \rightarrow \mathbf{S}^{n}$ and $P_{0}: \mathscr{U} \rightarrow \mathbf{S}^{n}$. Then $\left(\Omega, P_{0}\right)$ is a bounding pair if

$$
0 \leqslant P+P_{0}(\Delta A), \quad P \in \mathscr{N}, \Delta A \in \mathscr{U}
$$

and

$$
\Delta A^{\mathrm{T}} P+P \Delta A+(A+\Delta A)^{\mathrm{T}} P_{0}(\Delta A)+P_{0}(\Delta A)(A+\Delta A) \leqslant \Omega(P), \quad P \in \mathscr{N}, \Delta A \in \mathscr{U} .
$$

The following result, which is slightly stronger form of Theorem 3.1 of Reference [17] provides a bound for $J(\mathscr{U})$.

\section{Theorem 1}

Let $\left(\Omega, P_{0}\right)$ be a bounding pair and assume there exists $P \in \mathscr{N}$ satisfying

$$
A^{\mathrm{T}} P+P A+\Omega(P)+R \leqslant 0
$$

Then $(A+\Delta A, E)$ is detectable for all $\Delta A \in \mathscr{U}$ if and only if $A+\Delta A$ is asymptotically stable for all $\Delta A \in \mathscr{U}$. In this case,

$$
P_{\Delta A} \leqslant P+P_{0}(\Delta A), \quad \Delta A \in \mathscr{U},
$$

where $P_{\Delta A} \in \mathbf{N}^{n}$ is given by (3), and

$$
J(\mathscr{U}) \leqslant \mathscr{J}(\mathscr{U})
$$

where

$$
\mathscr{J}(\mathscr{U}) \triangleq \operatorname{tr} P V+\sup _{\Delta A \in \mathscr{U}} \operatorname{tr} P_{0}(\Delta A) V .
$$

\section{Remark 1}

If there exists $\bar{P}_{0} \in \mathbf{S}^{n}$ such that

$$
P_{0}(\Delta A) \leqslant \bar{P}_{0}, \quad \Delta A \in \mathscr{U},
$$

then

$$
\mathscr{J}(\mathscr{U}) \leqslant \overline{\mathscr{J}}
$$

where

$$
\overline{\mathscr{J}} \triangleq \operatorname{tr}\left[\left(P+\bar{P}_{0}\right) V\right]
$$

\section{Remark 2}

In Theorem 3.1 of Reference [17], inequality (6) appears as an equation. Inequality (6) is desirable since it permits the use of LMI techniques. 
A bounding pair $\left(\Omega, P_{0}\right)$ is parameter dependent if $P_{0}$ is not constant. Alternatively, a bounding pair $\left(\Omega, P_{0}\right)$ is parameter independent if $P_{0}$ is constant. In this case, $P_{0}(\Delta A)$ is replaced by $P_{0}$ and $\bar{P}_{0}=P_{0}$ so that $\overline{\mathscr{J}}=\mathscr{J}(\mathscr{U})=\operatorname{tr}\left[\left(P+P_{0}\right) V\right]$.

The remainder of the paper is concerned with the construction of bounding pairs $\left(\Omega, P_{0}\right)$. To construct a bounding pair $\left(\Omega, P_{0}\right)$ we must specify the set $\mathscr{N} \subseteq \mathbf{S}^{n}$ along with the functions $\Omega: \mathscr{N} \rightarrow \mathbf{S}^{n}$ and $P_{0}: \mathscr{U} \rightarrow \mathbf{S}^{n}$ that satisfy (4) and (5). No other assumptions on $\Omega$ and $P_{0}$ are required. To apply Theorem 1, however, requires the existence of a solution $P \in \mathscr{N}$ to inequality (6). LMI techniques will be used to obtain such solutions.

For a given bounding pair $\left(\Omega, P_{0}\right)$, the following immediate result yields an equivalent bounding pair $\left(\hat{\Omega}, \hat{P}_{0}\right)$.

\section{Proposition 1}

Let $\Omega: \mathscr{N} \subseteq \mathbf{S}^{n} \rightarrow \mathbf{S}^{n}, P \in \mathscr{N}$, and $P_{0}: \mathscr{U} \rightarrow \mathbf{S}^{n}$ satisfy (4)-(6), and let $\bar{P}_{0} \in \mathbf{S}^{n}$ satisfy (10). Furthermore, let $\overline{\hat{P}}_{0} \in \mathbf{S}^{n}$, and define $\hat{\mathcal{N}} \subseteq \mathbf{S}^{n}, \hat{\Omega}: \hat{\mathscr{N}} \rightarrow \mathbf{S}^{n}$ and $\hat{P}_{0}: \mathscr{U} \rightarrow \mathbf{S}^{n}$ by

$$
\begin{gathered}
\hat{\mathcal{N}} \triangleq \mathscr{N}+\bar{P}_{0}-\overline{\hat{P}}_{0}, \\
\hat{\Omega}(\hat{P}) \triangleq \Omega\left(\hat{P}-\bar{P}_{0}+\overline{\hat{P}}_{0}\right)-A^{\mathrm{T}}\left(\bar{P}_{0}-\overline{\hat{P}}_{0}\right)-\left(\bar{P}_{0}-\overline{\hat{P}}_{0}\right) A, \quad \hat{P} \in \hat{\mathcal{N}},
\end{gathered}
$$

and

$$
\hat{P}_{0}(\Delta A) \triangleq P_{0}(\Delta A)-\bar{P}_{0}+\overline{\hat{P}}_{0} .
$$

Then (4)-(6)_and (10) are satisfied with $\mathscr{N}, \Omega, P, P_{0}$, and $\bar{P}_{0}$ replaced by $\hat{\mathcal{N}}, \hat{\Omega}, P+\bar{P}_{0}-$ $\hat{\hat{P}}_{0}, \hat{P}_{0}$, and $\hat{\boldsymbol{P}}_{0}$. Furthermore, the bounding pairs $\left(\Omega, P_{0}\right)$ and $\left(\hat{\Omega}, \hat{P}_{0}\right)$ yield the same performance bound $\mathscr{J}(\mathscr{U})$.

Remark 3

If there exists $\Delta A \in \mathscr{U}$ such that $P_{0}(\Delta A)=0$, then (4) implies $P \geqslant 0$ for all $P \in \mathscr{N}$, and thus without loss of generality we can assume $\mathscr{N} \subseteq \mathbf{N}^{n}$.

\section{Remark 4}

Let $\left(\Omega, P_{0}\right)$ be a parameter-independent bounding pair with $\bar{P}_{0}=P_{0}$. Letting $\overline{\hat{P}}_{0}=0$ in Proposition 1 yields the equivalent parameter-independent bounding pair $(\hat{\Omega}, 0)$. Thus, without loss of generality, we can consider parameter-independent bounding pairs of the form $(\Omega, 0)$, where, by Remark 3, $\mathscr{N} \subseteq \mathbf{N}^{n}$.

In the following sections, $\mathscr{U}$ is given by either the parametric uncertainty set

$$
\mathscr{U}_{\mathrm{p}}(\mathscr{R})=\left\{\Delta A: \Delta A=\sum_{i=1}^{r} \delta_{i} A_{i}, \text { where }\left(\delta_{1}, \ldots, \delta_{r}\right) \in \mathscr{R}\right\},
$$

where $\mathscr{R} \subseteq \mathbf{R}^{r}$ and $A_{i} \in \mathbf{R}^{n \times n}, i=1, \ldots, r$, or the factored uncertainty set

$$
\mathscr{U}_{\mathrm{f}}(\mathscr{F})=\left\{\Delta A: \Delta A=B_{0} F C_{0} \text {, where } F \in \mathscr{F}\right\},
$$

where $\mathscr{F} \subseteq \mathbf{R}^{l_{1} \times l_{2}}, B_{0} \in \mathbf{R}^{n \times l_{1}}$ and $C_{0} \in \mathbf{R}^{l_{2} \times n}$. 
Note that the parametric uncertainty set $\mathscr{U}_{\mathrm{p}}(\mathscr{R})$ requires specification of the set $\mathscr{R}$, while the factored uncertainty set $\mathscr{U}_{\mathrm{f}}(\mathscr{F})$ requires specification of the set $\mathscr{F}$. These sets will be specified in later sections for each bounding pair that we consider.

Next we show that $\mathscr{U}_{\mathrm{p}}(\mathscr{R})$ is a special case of $\mathscr{U}_{\mathrm{f}}(\mathscr{F})$ for a special choice of $\mathscr{F}$. To show this, let $B_{i} \in \mathbf{R}^{n \times k_{i}}$ and $C_{i} \in \mathbf{R}^{k_{i} \times n}$ satisfy

$$
A_{i}=B_{i} C_{i}, \quad i=1, \ldots, r
$$

and define

$$
B_{0} \triangleq\left[B_{1} \cdots B_{r}\right] \in \mathbf{R}^{n \times k}, \quad C_{0} \triangleq\left[\begin{array}{c}
C_{1} \\
\vdots \\
C_{r}
\end{array}\right] \in \mathbf{R}^{k \times n},
$$

where $k \triangleq \sum_{i=1}^{r} k_{i}$. Then

$$
\Delta A=\sum_{i=1}^{r} \delta_{i} A_{i}=B_{0} F C_{0},
$$

where $F=\operatorname{diag}\left(\delta_{1} I_{k_{1}}, \ldots, \delta_{r} I_{k_{r}}\right) \in \mathbf{R}^{k \times k}$ so that $l_{1}=l_{2}=k$. Hence, with (18) and (19), it follows that

$$
\mathscr{U}_{\mathrm{f}}\left(\mathscr{F}_{\mathscr{R}}\right)=\mathscr{U}_{\mathrm{p}}(\mathscr{R})
$$

where $\mathscr{U}_{\mathrm{f}}\left(\mathscr{F}_{\mathscr{R}}\right)$ is the factored parametric uncertainty set, where

$$
\mathscr{F}_{\mathscr{R}} \triangleq\left\{F \in \mathbf{S}^{k}: F=\operatorname{diag}\left(\delta_{1} I_{k_{1}}, \ldots, \delta_{r} I_{k_{r}}\right),\left(\delta_{1}, \ldots, \delta_{r}\right) \in \mathscr{R}\right\} .
$$

\section{VERTEX LMIs FOR ROBUST PERFORMANCE}

In this section linear matrix inequalities are used to construct parameter-independent bounding pairs. For $\gamma>0$ define the polytopic uncertainty set

$$
\mathscr{U}_{\mathrm{p}}\left(\mathscr{R}_{\gamma}\right) \triangleq\left\{\Delta A: \Delta A=\sum_{i=1}^{r} \delta_{i} A_{i}, \text { where }\left|\delta_{i}\right| \leqslant \gamma, i=1, \ldots, r\right\},
$$

where

$$
\mathscr{R}_{\gamma} \triangleq\left\{\left(\delta_{1}, \ldots, \delta_{r}\right):\left|\delta_{i}\right| \leqslant \gamma, i=1, \ldots, r\right\} .
$$

With (18) and (19), the factored polytopic uncertainty set is given by

$$
\mathscr{U}_{\mathrm{f}}\left(\mathscr{F}_{\mathscr{R}_{\gamma}}\right)=\mathscr{U}_{\mathrm{p}}\left(\mathscr{R}_{\gamma}\right)
$$

where, with $\mathscr{R}=\mathscr{R}_{\gamma}$ in (22),

$$
\mathscr{F}_{\mathscr{R}_{\gamma}}=\left\{F \in \mathbf{S}^{k}: F=\operatorname{diag}\left(\delta_{1} I_{k_{1}}, \ldots, \delta_{r} I_{k_{r}}\right),\left|\delta_{i}\right| \leqslant \gamma, i=1, \ldots, r\right\} .
$$

\section{Lemma 1}

$P \in \mathbf{N}^{n}$ satisfies the $2^{r}$ LMIs

$$
A^{\mathrm{T}} P+P A \pm \gamma\left(A_{1}^{\mathrm{T}} P+P A_{1}\right) \pm \cdots \pm \gamma\left(A_{r}^{\mathrm{T}} P+P A_{r}\right)+R \leqslant 0
$$


Table III. LMI dimensions for continuous-time polytopic uncertainty bounds. For the linear and inverse families of bounds, $\alpha$ must be chosen separately.

\begin{tabular}{|c|c|c|c|c|}
\hline Bound & & Variables & Variable size & LMI dimension \\
\hline Vertex LMI & Prop. 2 & $P$ & $n^{2}$ & $2^{r} n^{2}$ \\
\hline $\begin{array}{l}\text { Shifted bounded real } \\
\text { Bounded real }\end{array}$ & Prop. 8 & ${ }_{P}^{P, N}, Y_{i}$ & $\begin{array}{l}n^{2}+2 \sum k_{i}^{2} \\
n^{2}\end{array}$ & $\begin{array}{l}(n+k)^{2}+2 \sum k_{i}^{2} \\
(n+k)^{2}\end{array}$ \\
\hline Shifted Popov & $\begin{array}{r}\text { Prop. } 11 \\
\text { Cor. } 1\end{array}$ & $\begin{array}{l}P, \bar{P}_{0}, \tilde{X}, Y, \tilde{N}, \tilde{H} \\
P, P_{i}, \tilde{X}, Y_{i}, \tilde{N}, \tilde{H}\end{array}$ & $\begin{array}{l}3 n^{2}+3 k^{2} \\
(2 r+1) n^{2}+3 k^{2}\end{array}$ & $\begin{array}{l}(n+k)^{2}+\left(2^{r+1}+1\right) n^{2} \\
(n+k)^{2}+(4 r+5) n^{2}\end{array}$ \\
\hline Popov & & $P, P_{i}, \tilde{N}, \tilde{H}$ & $(r+1) n^{2}+2 k^{2}$ & $(n+k)^{2}+(2 r+3) n^{2}$ \\
\hline Shifted linear & $\begin{array}{r}\text { Prop. } 15 \\
\text { Cor. } 2\end{array}$ & $\begin{array}{l}P, N, Y \\
P, N, Y_{i}\end{array}$ & $\begin{array}{l}(r+2) n^{2} \\
(r+2) n^{2}\end{array}$ & $\begin{array}{l}\left(2^{r}+2\right) n^{2} \\
(2 r+2) n^{2}\end{array}$ \\
\hline Linear & & $P$ & & $2 n^{2}$ \\
\hline Shifted inverse & $\begin{array}{r}\text { Prop. } 18 \\
\text { Remark } 12\end{array}$ & 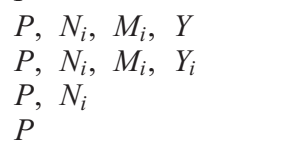 & $\begin{array}{l}(2 r+2) n^{2} \\
(3 r+1) n^{2} \\
(r+1) n^{2} \\
n^{2}\end{array}$ & $\begin{array}{l}(r+1)^{2} n^{2}+\left(2^{r}+1\right) n^{2} \\
(r+1)^{2} n^{2}+(2 r+1) n^{2} \\
(r+1)^{2} n^{2}+n^{2} \\
(r+1)^{2} n^{2}+n^{2}\end{array}$ \\
\hline
\end{tabular}

if and only if $P$ satisfies

$$
(A+\Delta A)^{\mathrm{T}} P+P(A+\Delta A)+R \leqslant 0, \quad \Delta A \in \mathscr{U}_{\mathrm{p}}\left(\mathscr{R}_{\gamma}\right) .
$$

The following result shows that the set of solutions to the $2^{r}$ vertex LMIs (27) gives rise to a parameter-independent bounding pair $\left(\Omega_{\mathrm{LMI}}, 0\right)$. Define

$$
\mathscr{P} \triangleq\left\{P \in \mathbf{N}^{n}: P \text { satisfies }(27)\right\} .
$$

\section{Proposition 2}

Let $\mathscr{U}=\mathscr{U}_{\mathrm{p}}\left(\mathscr{R}_{\gamma}\right)$ and define $\Omega_{\mathrm{LMI}}: \mathscr{P} \rightarrow \mathbf{N}^{n}$ by

$$
\Omega_{\mathrm{LMI}}(P) \triangleq-R-A^{\mathrm{T}} P-P A .
$$

Then $\left(\Omega_{\mathrm{LMI}}, 0\right)$ is a bounding pair. Furthermore, $J\left(\mathscr{U}_{\mathrm{p}}\left(\mathscr{R}_{\gamma}\right)\right) \leqslant \operatorname{tr} P V$ for all $P \in \mathscr{P}$.

The next result shows that every bound $\mathscr{J}(\mathscr{U})$ obtainable from a parameter-independent bounding pair $(\Omega, 0)$ is also obtainable from vertex LMIs.

\section{Proposition 3}

Let $\mathscr{U}=\mathscr{U}_{\mathrm{p}}\left(\mathscr{R}_{\gamma}\right)$, let $(\Omega, 0)$ be a bounding pair, where $\Omega: \mathscr{N} \subseteq \mathbf{N}^{n} \rightarrow \mathbf{S}^{n}$, and assume there exists $P \in \mathscr{N}$ satisfying (6). Then $P$ satisfies (27).

Propositions 2 and 3 show that there is an equivalence between the performance bounds obtainable from vertex LMIs and the performance bounds obtainable from parameterindependent bounding pairs $(\Omega, 0)$. This equivalence does not suggest, however, that parameterindependent bounding pairs $(\Omega, 0)$ are of no interest. Rather, as can be seen in Table III, the bounding pairs $(\Omega, 0)$ may entail LMIs that are of lower dimensionality than vertex LMIs. With this motivation in mind, we turn our attention to the construction of parameter-independent and parameter-dependent bounding pairs. 


\section{SHIFTED BOUNDED REAL BOUND}

Define $\mathscr{F}_{\mathrm{BR}}(M) \subset \mathbf{R}^{l_{1} \times l_{2}}$ by

$$
\mathscr{F}_{\mathrm{BR}}(M) \triangleq\left\{F \in \mathbf{R}^{l_{1} \times l_{2}}: F^{\mathrm{T}} F \leqslant M\right\},
$$

where $M \in \mathbf{N}^{l_{2}}$. The following result concerns the classical bounded real bound [3,5].

Proposition 4

Let $\mathscr{U}=\mathscr{U}_{\mathrm{f}}\left(\mathscr{F}_{\mathrm{BR}}(M)\right), \mathscr{N}=\mathbf{N}^{n}$, and

$$
\Omega(P)=P B_{0} B_{0}^{\mathrm{T}} P+C_{0}^{\mathrm{T}} M C_{0} .
$$

Then $(\Omega, 0)$ is a bounding pair.

Next, define

$$
\mathscr{F}_{\mathrm{BRs}}(M, N) \triangleq\left\{F \in \mathbf{R}^{l_{1} \times l_{2}}:(F+N)^{\mathrm{T}}(F+N) \leqslant M\right\},
$$

where $M \in \mathbf{N}^{l_{2}}$ and $N \in \mathbf{R}^{l_{1} \times l_{2}}$. Note that $\mathscr{F}_{\mathrm{BRs}}(M, 0)=\mathscr{F}_{\mathrm{BR}}(M)$. The following result concerns the shifted bounded real bound [18].

Proposition 5

Let $\mathscr{U}=\mathscr{U}_{\mathrm{f}}\left(\mathscr{F}_{\mathrm{BRs}}(M, N)\right), \mathscr{N}=\mathbf{N}^{n}$, and

$$
\Omega(P)=P B_{0} B_{0}^{\mathrm{T}} P-\left(B_{0} N C_{0}\right)^{\mathrm{T}} P-P B_{0} N C_{0}+C_{0}^{\mathrm{T}} M C_{0} .
$$

Then $(\Omega, 0)$ is a bounding pair.

The shifted bounded real bound inequality is given by (6) with $\Omega$ given by (33), which has the form

$$
\left(A-B_{0} N C_{0}\right)^{\mathrm{T}} P+P\left(A-B_{0} N C_{0}\right)+P B_{0} B_{0}^{\mathrm{T}} P+C_{0}^{\mathrm{T}} M C_{0}+R \leqslant 0 .
$$

Remark 5

Note that

$$
\begin{aligned}
A+\mathscr{U}_{\mathrm{f}}\left(\mathscr{F}_{\mathrm{BRs}}(M, N)\right) & =\left\{A+B_{0} F C_{0}: F \in \mathscr{F}_{\mathrm{BRs}}(M, N)\right\} \\
& =\left\{A+B_{0} F C_{0}:(F+N)^{\mathrm{T}}(F+N) \leqslant M\right\} \\
& =\left\{A+B_{0}(\hat{F}-N) C_{0}: \hat{F}^{\mathrm{T}} \hat{F} \leqslant M\right\} \\
& =\left\{A_{\mathrm{s}}+B_{0} \hat{F} C_{0}: \hat{F}^{\mathrm{T}} \hat{F} \leqslant M\right\} \\
& =A_{\mathrm{s}}+\mathscr{U}_{\mathrm{f}}\left(\mathscr{F}_{\mathrm{BR}}(M)\right),
\end{aligned}
$$

where $A_{\mathrm{s}} \triangleq A-B_{0} N C_{0}$. This identity suggests that the shifted bounded real bound is not more general than the bounded real bound. However, this is definitely not the case. Rather, the shifted bounded real bound has the form of the bounded real bound for a shifted nominal dynamics 
matrix $A_{\mathrm{S}}$ that is different from the original nominal dynamics matrix $A$. The numerical results in Section 8 show that, for the examples considered, the shifted bounded real bound is markedly less conservative than the standard bounded real bound.

\section{Remark 6}

Other factorizations can be used in place of (32). In particular, [18] uses a factorization of the form

$$
\hat{\mathscr{F}}_{\mathrm{BRs}}\left(M_{\mathrm{s}}, N\right) \triangleq\left\{F \in \mathbf{R}^{l_{1} \times l_{2}}:\left(F C_{0}+N\right)^{\mathrm{T}}\left(F C_{0}+N\right) \leqslant M_{\mathrm{s}}\right\},
$$

where $N$ and $M_{\mathrm{s}}$ are chosen to have appropriate dimension. Example 3 in Section 8 uses a factorization of this form for the shifted bounded-real bound.

Next we apply the shifted bounded real bound to the factored polytopic uncertainty set $\mathscr{U}_{\mathrm{f}}\left(\mathscr{F}_{\mathscr{R}_{\gamma}}\right)=\mathscr{U}_{\mathrm{p}}\left(\mathscr{R}_{\gamma}\right)$ with $B_{0}, C_{0}$ given by (18) and (19), so that $F=\operatorname{diag}\left(\delta_{1} I_{k_{1}}, \ldots, \delta_{r} I_{k_{r}}\right) \in \mathscr{F}_{\mathscr{R}_{\gamma}}$. Note that if $\gamma^{2} I \leqslant M$ then $\mathscr{F}_{\mathscr{R}_{\gamma}} \subseteq \mathscr{F}_{\mathrm{BR}}(M)$. Now let $N=\operatorname{diag}\left(N_{1}, \ldots, N_{r}\right) \in \mathbf{R}^{k \times k}$, where $N_{i} \in$ $\mathbf{R}^{k_{i} \times k_{i}}, i=1, \ldots, r$. Then

$$
\begin{aligned}
\mathscr{F}_{\operatorname{BRs} \mathscr{R}}(M, N) & \triangleq \mathscr{F}_{\mathrm{BRs}}(M, N) \cap \mathscr{F}_{\mathscr{R}} \\
& =\left\{F=\operatorname{diag}\left(\delta_{1} I_{k_{1}}, \ldots, \delta_{r} I_{k_{r}}\right):(F+N)^{\mathrm{T}}(F+N) \leqslant M,\left(\delta_{1}, \ldots, \delta_{r}\right) \in \mathscr{R}\right\} .
\end{aligned}
$$

\section{Proposition 6}

Let $M$ be given by

$$
M=N^{\mathrm{T}} N+\gamma^{2} I+Y,
$$

where $Y \triangleq \operatorname{diag}\left(Y_{1}, \ldots, Y_{r}\right)$, and $Y_{i} \in \mathbf{N}^{k_{i}}, i=1, \ldots, r$, satisfies

$$
\delta_{i}\left(N_{i}+N_{i}^{\mathrm{T}}\right) \leqslant Y_{i}, \quad\left|\delta_{i}\right| \leqslant \gamma, \quad i=1, \ldots, r .
$$

Then

$$
\mathscr{F}_{\mathscr{R}_{\gamma}} \subseteq \mathscr{F}_{\mathrm{BRs}}(M, N) .
$$

With $M$ given by (35), (34) becomes

$$
\left(A-B_{0} N C_{0}\right)^{\mathrm{T}} P+P\left(A-B_{0} N C_{0}\right)+P B_{0} B_{0}^{\mathrm{T}} P+C_{0}^{\mathrm{T}}\left(N^{\mathrm{T}} N+\gamma^{2} I+Y\right) C_{0}+R \leqslant 0 .
$$

The next proposition gives two choices of $Y_{i}$ that satisfy (36).

\section{Proposition 7}

Let $Y_{i} \in \mathbf{N}^{k_{i}}, i=1, \ldots, r$, and consider the conditions

$$
Y_{i}=\gamma\left|N_{i}+N_{i}^{\mathrm{T}}\right|, \quad i=1, \ldots, r,
$$

and

$$
-Y_{i} \leqslant \gamma\left(N_{i}+N_{i}^{\mathrm{T}}\right) \leqslant Y_{i}, \quad i=1, \ldots, r .
$$

Then $(39) \Rightarrow(40) \Leftrightarrow(36)$.

Next, we formulate an LMI to obtain a feasible solution $P \in \mathbf{N}^{n}$ to the shifted bounded real inequality (34) along with $M$ and $N$. The following result follows from the equivalence of (36) 
and (40) as well as by using Schur complements to rewrite (38). Let $N=\operatorname{diag}\left(N_{1}, \ldots, N_{r}\right)$ and $Y=\operatorname{diag}\left(Y_{1}, \ldots, Y_{r}\right)$.

Proposition 8

Let $\mathscr{U}=\mathscr{U}_{\mathrm{f}}\left(\mathscr{F}_{\mathscr{R}_{\gamma}}\right)$ and let $P \in \mathbf{N}^{n}, N \in \mathbf{R}^{k \times k}$, and $Y \in \mathbf{S}^{n}$. Then $P, N, Y$ satisfy (40) and

$$
\left[\begin{array}{cc}
A^{\mathrm{T}} P+P A+C_{0}^{\mathrm{T}}\left(\gamma^{2} I+Y\right) C_{0}+R & P B_{0}-C_{0}^{\mathrm{T}} N^{\mathrm{T}} \\
B_{0}^{\mathrm{T}} P-N C_{0} & -I
\end{array}\right] \leqslant 0
$$

if and only if $P, N, Y$ satisfy (36) and (38).

\section{Remark 7}

The LMI (41) is a special case of (24) in Reference [15] with

$$
\hat{Q}=C_{0}^{\mathrm{T}}\left(\gamma^{2} I+Y\right) C_{0}+R, \quad \hat{S}=C_{0}^{\mathrm{T}} N^{\mathrm{T}}, \quad \hat{R}=-I, \quad B=B_{0}, \quad C=I .
$$

\section{SHIFTED POPOV BOUND}

Let $l_{1}=l_{2}=k$ and define $\mathscr{H}_{\mathrm{P}} \subset \mathbf{S}^{k}, \mathscr{H}_{\mathrm{P}} \subset \mathbf{P}^{k}$ and $\mathscr{N}_{\mathrm{P}} \subset \mathbf{R}^{k \times k}$ by

$$
\begin{gathered}
\mathscr{F}_{\mathrm{P}} \subseteq\left\{F \in \mathbf{S}^{k}: M_{\mathrm{L}} \leqslant F \leqslant M_{\mathrm{U}}\right\}, \\
\mathscr{H}_{\mathrm{P}} \triangleq\left\{H \in \mathbf{P}^{k}: H F=F H, F \in \mathscr{F}_{\mathrm{P}}\right\}, \\
\mathscr{N}_{\mathrm{P}} \triangleq\left\{N \in \mathbf{R}^{k \times k}: N^{\mathrm{T}} M_{\mathrm{L}}=M_{\mathrm{L}} N, N^{\mathrm{T}} F=F N, F \in \mathscr{F} \mathrm{P}\right\},
\end{gathered}
$$

where $M_{\mathrm{L}}, M_{\mathrm{U}} \in \mathbf{S}^{k}$ are such that

$$
M \triangleq M_{\mathrm{U}}-M_{\mathrm{L}}>0 .
$$

The following result concerns the Popov bound [17].

Proposition 9

Let $\mathscr{U}=\mathscr{U}_{\mathrm{f}}\left(\mathscr{F}_{\mathrm{P}}\right), \mathscr{N}=\mathbf{N}^{n}, N \in \mathscr{N}_{\mathrm{P}}$ and $H \in \mathscr{H}_{\mathrm{P}}$. Assume that

$$
R_{0} \triangleq\left(H M^{-1}-N C_{0} B_{0}\right)+\left(H M^{-1}-N C_{0} B_{0}\right)^{\mathrm{T}}>0
$$

and let

$$
\begin{aligned}
\Omega(P)= & \left(H C_{0}+N C_{0} \tilde{A}_{\mathrm{P}}+B_{0}^{\mathrm{T}} P\right)^{\mathrm{T}} R_{0}^{-1}\left(H C_{0}+N C_{0} \tilde{A}_{\mathrm{P}}+B_{0}^{\mathrm{T}} P\right) \\
& +\left(B_{0} M_{\mathrm{L}} C_{0}\right)^{\mathrm{T}} P+P B_{0} M_{\mathrm{L}} C_{0},
\end{aligned}
$$

where $\tilde{A}_{\mathrm{P}} \triangleq A+B_{0} M_{\mathrm{L}} C_{0}$, and

$$
P_{0}(\Delta A)=C_{0}^{\mathrm{T}}\left(F-M_{\mathrm{L}}\right) N C_{0} .
$$

Then $\left(\Omega, P_{0}\right)$ is a bounding pair. 
Remark 8

If $\mu \in \mathbf{S}^{k}$ satisfies $\mu \geqslant\left(F-M_{\mathrm{L}}\right) N$ for all $F \in \mathscr{F}$ P, then $\bar{P}_{0}=C_{0}^{\mathrm{T}} \mu C_{0}$ satisfies (10).

The following result concerns the shifted Popov bound [19].

\section{Proposition 10}

Let $\mathscr{U}=\mathscr{U}_{\mathrm{f}}(\mathscr{F} \mathrm{P})$, let $X \in \mathbf{R}^{k \times k}$ and $Y \in \mathbf{S}^{n}$ satisfy

$$
B_{0} X^{\mathrm{T}}\left(F-M_{\mathrm{L}}\right) C_{0}+C_{0}^{\mathrm{T}}\left(F-M_{\mathrm{L}}\right) X B_{0}^{\mathrm{T}} \leqslant Y, \quad F \in \mathscr{F} \mathrm{P}
$$

let $\mathscr{N}=\mathbf{N}^{n}, N \in \mathscr{N}_{\mathrm{P}}$, and $H \in \mathscr{H}_{\mathrm{P}}$, let $R_{0}$ be given by (46), define

$$
\begin{aligned}
\Omega(P)= & \left(H C_{0}+N C_{0} \tilde{A}_{\mathrm{P}}+B_{0}^{\mathrm{T}} P-X B_{0}^{\mathrm{T}}\right)^{\mathrm{T}} R_{0}^{-1}\left(H C_{0}+N C_{0} \tilde{A}_{\mathrm{P}}+B_{0}^{\mathrm{T}} P-X B_{0}^{\mathrm{T}}\right) \\
& +\left(B_{0} M_{\mathrm{L}} C_{0}\right)^{\mathrm{T}} P+P B_{0} M_{\mathrm{L}} C_{0}+Y,
\end{aligned}
$$

and let $P_{0}(\Delta A)$ be given by (48). Then $\left(\Omega, P_{0}\right)$ is a bounding pair.

The shifted Popov bound inequality is given by (6) with $\Omega$ given by (50), which has the form

$$
\begin{aligned}
& \left(A+B_{0} M_{\mathrm{L}} C_{0}\right)^{\mathrm{T}} P+P\left(A+B_{0} M_{\mathrm{L}} C_{0}\right)+\left(H C_{0}+N C_{0} \tilde{A}_{\mathrm{P}}+B_{0}^{\mathrm{T}} P-X B_{0}^{\mathrm{T}}\right)^{\mathrm{T}} \\
& \times R_{0}^{-1}\left(H C_{0}+N C_{0} \tilde{A}_{\mathrm{P}}+B_{0}^{\mathrm{T}} P-X B_{0}^{\mathrm{T}}\right)+Y+R \leqslant 0 .
\end{aligned}
$$

Remark 9

Setting $X=0$ and $Y=0$ in Proposition 10 yields Proposition 9.

Next, define $\mathscr{H}_{\mathrm{Pd}} \subset \mathbf{P}^{k}, \mathscr{N}_{\mathrm{Pd}} \subset \mathbf{S}^{k}$ and $\hat{I}_{1}, \ldots, \hat{I}_{r} \in \mathbf{S}^{k}$ by

$$
\begin{aligned}
& \mathscr{H}_{\mathrm{Pd}} \triangleq\left\{H \in \mathbf{P}^{k}: H=\operatorname{diag}\left(H_{1}, \ldots, H_{r}\right), H_{i} \in \mathbf{P}^{k_{i}}, i=1, \ldots, r\right\}, \\
& \mathscr{N}_{\mathrm{Pd}} \triangleq\left\{N \in \mathbf{S}^{k}: N=\operatorname{diag}\left(N_{1}, \ldots, N_{r}\right), N_{i} \in \mathbf{S}^{k_{i}}, i=1, \ldots, r\right\},
\end{aligned}
$$

and

$$
\hat{I}_{i} \triangleq \operatorname{diag}\left(0_{k_{1}}, \ldots, 0_{k_{i-1}}, I_{k_{i}}, 0_{k_{i+1}}, \ldots, 0_{k_{r}}\right),
$$

where $k=\sum_{i=1}^{r} k_{i}$. Let $-M_{\mathrm{L}}=M_{\mathrm{U}}=\gamma I$ and let $\mathscr{F}_{P} \subseteq \mathscr{F}_{\mathscr{R}}$. Then $\mathscr{F}_{\mathrm{P}}=\mathscr{F}_{\mathscr{R}_{\gamma}}$. The following result provides an LMI satisfying (49) and (6) with $\mathscr{U}=\mathscr{U}_{\mathrm{f}}\left(\mathscr{F}_{\mathscr{R}_{\gamma}}\right)=\mathscr{U}_{\mathrm{p}}\left(\mathscr{R}_{\gamma}\right)$ and with $\Omega$ given by (50).

\section{Proposition 11}

Let $\mathscr{U}=\mathscr{U}_{\mathrm{p}}\left(\mathscr{R}_{\gamma}\right)$, and let $\tilde{\mathscr{N}}$ denote the set of $(P, X, Y, N, H) \in \mathbf{N}^{n} \times \mathbf{R}^{k \times k} \times \mathbf{S}^{n} \times \mathscr{N}_{P \gamma} \times \mathscr{H}_{\mathrm{Pd}}$ satisfying

$$
\left[\begin{array}{cc}
A_{\mathrm{P}}^{\mathrm{T}} P+P A_{\mathrm{P}}+Y+R & C_{0}^{\mathrm{T}} H+\tilde{A}_{\mathrm{P}}^{\mathrm{T}} C_{0}^{\mathrm{T}} N+P B_{0}-B_{0} X^{\mathrm{T}} \\
H C_{0}+N C_{0} \tilde{A}_{\mathrm{P}}+B_{0}^{\mathrm{T}} P-X B_{0}^{\mathrm{T}} & N C_{0} B_{0}+B_{0}^{\mathrm{T}} C_{0}^{\mathrm{T}} N-\gamma^{-1} H
\end{array}\right]<0
$$


and the $2^{r+1}$ LMIs

$$
\begin{aligned}
& \pm \gamma\left(B_{0} X^{\mathrm{T}} C_{0}+C_{0}^{\mathrm{T}} X B_{0}^{\mathrm{T}}\right) \pm \gamma\left(B_{0} X^{\mathrm{T}} \hat{I}_{1} C_{0}+C_{0}^{\mathrm{T}} \hat{I}_{1} X B_{0}^{\mathrm{T}}\right) \\
& \pm \cdots \pm \gamma\left(B_{0} X^{\mathrm{T}} \hat{I}_{r} C_{0}+C_{0}^{\mathrm{T}} \hat{I}_{r} X B_{0}^{\mathrm{T}}\right) \leqslant Y .
\end{aligned}
$$

Then (6), with $\Omega$ given by (50), and (49) are satisfied for all $(P, X, Y, N, H) \in \tilde{\mathscr{N}}$. Furthermore, if $\bar{P}_{0} \in \mathbf{S}^{n}$ satisfies the $2^{r+1}$ LMIs

$$
\gamma C_{0}^{\mathrm{T}}\left( \pm I \pm \hat{I}_{1} \pm \hat{I}_{2} \pm \cdots \pm \hat{I}_{r}\right) N C_{0} \leqslant \bar{P}_{0}
$$

then (10) is satisfied.

Corollary 1

Let $X \in \mathbf{R}^{k \times k}$ and let $Y_{1}, \ldots, Y_{r+1} \in \mathbf{S}^{n}$ satisfy

$$
\begin{gathered}
-Y_{i} \leqslant \gamma B_{0} X^{\mathrm{T}} \hat{I}_{i} C_{0}+\gamma C_{0}^{\mathrm{T}} X \hat{\boldsymbol{I}}_{i} B_{0}^{\mathrm{T}} \leqslant Y_{i}, \quad i=1, \ldots, r, \\
-Y_{r+1} \leqslant \gamma B_{0} X^{\mathrm{T}} C_{0}+\gamma C_{0}^{\mathrm{T}} X B_{0}^{\mathrm{T}} \leqslant Y_{r+1},
\end{gathered}
$$

let $N \in \mathscr{N}_{\mathrm{P} \gamma}$ and let $P_{1}, \ldots, P_{r+1} \in \mathbf{S}^{n}$ satisfy

$$
\begin{gathered}
-P_{i} \leqslant \gamma C_{0}^{\mathrm{T}} \hat{I}_{i} N C_{0} \leqslant P_{i}, \quad i=1, \ldots, r, \\
-P_{r+1} \leqslant \gamma C_{0}^{\mathrm{T}} N C_{0} \leqslant P_{r+1} .
\end{gathered}
$$

Then $Y=\sum_{i=1}^{r+1} Y_{i}$ satisfies (49). Finally, let $P_{0}(\Delta A)$ be given by (48). Then $\bar{P}_{0}=\sum_{i=1}^{r+1} P_{i}$ satisfies (10).

\section{SHIFTED LINEAR BOUND}

In this section we consider the linear bound [22-24].

\section{Proposition 12}

Let $\mathscr{U}=\mathscr{U}_{\mathrm{p}}\left(\mathscr{R}_{\gamma}\right), \alpha>0$ and $\mathscr{N}=\mathbf{N}^{n}$, and define

$$
\Omega(P)=\alpha r P+\frac{\gamma^{2}}{\alpha} \sum_{i=1}^{r} A_{i}^{\mathrm{T}} P A_{i} .
$$

Then $(\Omega, 0)$ is a bounding pair.

Next, the shifted linear bound is obtained.

\section{Proposition 13}

Let $\mathscr{U}=\mathscr{U}_{\mathrm{p}}\left(\mathscr{R}_{\gamma}\right)$ and $\alpha>0$, let $N_{1}, \ldots, N_{r}, Y \in \mathbf{S}^{n}$ satisfy

$$
\sum_{i=1}^{r} \delta_{i}\left(A_{i}^{\mathrm{T}} N_{i}+N_{i}^{\mathrm{T}} A_{i}\right) \leqslant Y, \quad\left|\delta_{i}\right| \leqslant \gamma, \quad i=1, \ldots r,
$$

and define $\mathscr{N}=\mathbf{N}^{n} \cap\left(\bigcap_{i=1}^{r}\left[\mathbf{N}^{n}+N_{i}\right]\right)$ and

$$
\Omega(P)=\sum_{i=1}^{r}\left[\alpha\left(P-N_{i}\right)+\frac{\gamma^{2}}{\alpha} A_{i}^{\mathrm{T}}\left(P-N_{i}\right) A_{i}\right]+Y .
$$

Then $(\Omega, 0)$ is a bounding pair. 
Remark 10

Setting $N_{1}=\cdots=N_{r}=0$ and $Y=0$ in Proposition 13 yields Proposition 12 .

The shifted linear bound inequality is given by (6) with $\Omega$ given by (63), which has the form

$$
A^{\mathrm{T}} P+P A+\sum_{i=1}^{r}\left[\alpha\left(P-N_{i}\right)+\frac{\gamma^{2}}{\alpha} A_{i}^{\mathrm{T}}\left(P-N_{i}\right) A_{i}\right]+Y+R \leqslant 0 .
$$

The next result provides a method for computing $P$ satisfying

$$
A^{\mathrm{T}} P+P A+\sum_{i=1}^{r}\left[\alpha\left(P-N_{i}\right)+\frac{\gamma^{2}}{\alpha} A_{i}^{\mathrm{T}}\left(P-N_{i}\right) A_{i}\right]+Y+R=0 .
$$

Proposition 14

Let $\alpha>0, N_{1}, \ldots, N_{r} \in \mathbf{S}^{n}$, and $Y=\gamma \sum_{i=1}^{r}\left|A_{i}^{\mathrm{T}} N_{i}+N_{i}^{\mathrm{T}} A_{i}\right|$. Suppose

$$
\mathscr{A} \triangleq\left(A+\frac{\alpha \tau}{2} I\right) \oplus\left(A+\frac{\alpha r}{2} I\right)+\frac{\gamma^{2}}{\alpha} \sum_{i=1}^{r}\left(A_{i} \otimes A_{i}\right)
$$

is invertible. Then (65) has the unique solution

$$
P=-\operatorname{vec}^{-1}\left(\mathscr{A}^{-\mathrm{T}} \operatorname{vec} R_{0}\right),
$$

where

$$
R_{0} \triangleq \sum_{i=1}^{r}\left[\gamma\left|A_{i}^{\mathrm{T}} N_{i}+N_{i} A_{i}\right|-\alpha N_{i}-\frac{\gamma^{2}}{\alpha} A_{i}^{\mathrm{T}} N_{i} A_{i}\right]+R
$$

If, in addition, $\mathscr{A}$ is asymptotically stable and $R_{0}$ is non-negative definite, then $P \geqslant 0$.

\section{Remark 11}

The last statement of Proposition 14 follows from techniques used in Reference [26].

Proposition 15

Let $\mathscr{U}=\mathscr{U}_{\mathrm{p}}\left(\mathscr{R}_{\gamma}\right)$, let $\alpha>0$, and let $\tilde{\mathcal{N}}$ denote the set of $\left(P, N_{1}, \ldots, N_{r}, Y\right) \in \mathbf{N}^{n} \times\left(\mathbf{S}^{n}\right)^{r+1}$ satisfying

$$
A^{\mathrm{T}} P+P A+\sum_{i=1}^{r}\left[\alpha\left(P-N_{i}\right)+\frac{\gamma^{2}}{\alpha} A_{i}^{\mathrm{T}}\left(P-N_{i}\right) A_{i}\right]+Y+R \leqslant 0
$$

and the $2^{r}$ LMIs

$$
\pm \gamma\left(A_{i}^{\mathrm{T}} N_{1}+N_{1} A_{1}\right) \pm \cdots \pm \gamma\left(A_{r}^{\mathrm{T}} N_{r}+N_{r} A_{r}\right) \leqslant Y .
$$

Then (62) and (63) are satisfied for all $\left(P, N_{1}, \ldots, N_{r}, Y\right) \in \tilde{\mathscr{N}}$.

Letting $N_{1}=\cdots=N_{r}=N$ in Proposition 13 yields the following specialization of the shifted linear bound.

Corollary 2

Let $\alpha>0$ and let $N, Y_{1}, \ldots, Y_{r} \in \mathbf{S}^{n}$ satisfy

$$
\delta_{i}\left(A_{i}^{\mathrm{T}} N+N A_{i}\right) \leqslant Y_{i}, \quad\left|\delta_{i}\right| \leqslant \gamma, \quad i=1, \ldots, r,
$$


and define $\mathscr{N}=\mathbf{N}^{n} \cap\left(\mathbf{N}^{n}+N\right)$ and

$$
\Omega(P)=\alpha r(P-N)+\sum_{i=1}^{r} \frac{\gamma^{2}}{\alpha} A_{i}^{\mathrm{T}}(P-N) A_{i}+\sum_{i=1}^{r} Y_{i} .
$$

Then $(\Omega, 0)$ is a bounding pair.

\section{SHIFTED INVERSE BOUND}

The following result concerns the inverse bound [4].

Proposition 16

Let $\mathscr{U}=\mathscr{U}_{\mathrm{p}}\left(\mathscr{R}_{\gamma}\right), \alpha>0$ and $\mathscr{N}=\mathbf{P}^{n}$, and define

$$
\Omega(P)=\alpha \gamma r P+\frac{\gamma}{4 \alpha} \sum_{i=1}^{r}\left(A_{i}^{\mathrm{T}} P+P A_{i}\right) P^{-1}\left(A_{i}^{\mathrm{T}} P+P A_{i}\right) .
$$

Then $(\Omega, 0)$ is a bounding pair.

The inverse bound inequality, which is given by (6) with $\Omega$ given by (72), has the form

$$
A^{\mathrm{T}} P+P A+\alpha \gamma r P+\frac{\gamma}{4 \alpha} \sum_{i=1}^{r}\left(A_{i}^{\mathrm{T}} P+P A_{i}\right) P^{-1}\left(A_{i}^{\mathrm{T}} P+P A_{i}\right)+R \leqslant 0 .
$$

Equation (73) can be written as

$$
\tilde{A}_{\text {inv }}^{\mathrm{T}} P+P \tilde{A}_{\mathrm{inv}}+\frac{\gamma}{4 \alpha} \sum_{i=1}^{r}\left(A_{i}^{\mathrm{T}} P A_{i}+P A_{i} P^{-1} A_{i}^{\mathrm{T}} P\right)+R \leqslant 0,
$$

where $\tilde{A}_{\text {inv }} \triangleq A+\alpha r \frac{\gamma}{2} I+\frac{\gamma}{4 \alpha} \sum_{i=1}^{r} A_{i}^{2}$.

Next, the shifted inverse bound is obtained.

Proposition 17

Let $\mathscr{U}=\mathscr{U}_{\mathrm{p}}\left(\mathscr{R}_{\gamma}\right)$ and $\alpha>0$, and let $M_{1 i}, M_{2 i} \in \mathbf{R}^{n \times n}, i=1, \ldots, r$, and $Y \in \mathbf{S}^{n}$ satisfy

$$
\sum_{i=1}^{r} \frac{\delta_{i}}{2}\left[A_{i}^{\mathrm{T}}\left(M_{1 i}+M_{2 i}^{\mathrm{T}}\right)+\left(M_{2 i}+M_{1 i}^{\mathrm{T}}\right) A_{i}\right] \leqslant Y, \quad\left|\delta_{i}\right| \leqslant \gamma, \quad i=1, \ldots, r .
$$

Let $N_{1}, \ldots, N_{r} \in \mathbf{S}^{n}$, and define

$$
\mathscr{N}=\mathbf{N}^{n} \cap\left(\bigcap_{i=1}^{r}\left[\mathbf{P}^{n}+N_{i}\right]\right)
$$

and

$$
\begin{aligned}
\Omega(P)= & \sum_{i=1}^{r}\left(\frac{\gamma}{4 \alpha}\left[A_{i}^{\mathrm{T}}\left(P-M_{1 i}\right)+\left(P-M_{2 i}\right) A_{i}\right]\left(P-N_{i}\right)^{-1}\right. \\
& \left.\times\left[A_{i}^{\mathrm{T}}\left(P-M_{1 i}\right)+\left(P-M_{2 i}\right) A_{i}\right]^{\mathrm{T}}+\alpha \gamma\left(P-N_{i}\right)\right)+Y .
\end{aligned}
$$

Then $(\Omega, 0)$ is a bounding pair. 
Substituting $\Omega(P)$ into (6) yields the shifted inverse bound inequality

$$
\begin{aligned}
& A^{\mathrm{T}} P+P A+\sum_{i=1}^{r}\left(\frac{\gamma}{4 \alpha}\left[A_{i}^{\mathrm{T}}\left(P-M_{1 i}\right)+\left(P-M_{2 i}\right) A_{i}\right]\left(P-N_{i}\right)^{-1}\right. \\
& \left.\quad \times\left[A_{i}^{\mathrm{T}}\left(P-M_{1 i}\right)+\left(P-M_{2 i}\right) A_{i}\right]^{\mathrm{T}}-\alpha \gamma N_{i}\right)+\alpha \gamma r P+Y+R \leqslant 0 .
\end{aligned}
$$

Let $M_{i 1}=M_{2 i}=M_{i} \in \mathbf{S}^{n}, i=1, \ldots, r$. The next result uses LMIs to find $P \in \mathbf{P}^{n}$ and $M_{1}, \ldots$, $M_{r}, N_{1}, \ldots, N_{r}$ and $Y \in \mathbf{S}^{n}$ satisfying (75) and (78).

\section{Proposition 18}

Let $\mathscr{U}=\mathscr{U}_{\mathrm{p}}\left(\mathscr{R}_{\gamma}\right)$ and let $\alpha>0$. Let $\tilde{N}$ denote the set of $\left(P, N_{1}, \ldots, N_{r}, M_{1}, \ldots, M_{r}, Y\right) \in \mathbf{N}^{n} \times$ $\left(\mathbf{S}^{n}\right)^{2 r+1}$ satisfying

$$
\left[\begin{array}{cccc}
A^{\mathrm{T}} P+P A+\alpha \gamma \sum_{i=1}^{r}\left(P-N_{i}\right)+Y+R & A_{1}^{\mathrm{T}}\left(P-M_{1}\right)+\left(P-M_{1}\right) A_{1} & \cdots & A_{r}^{\mathrm{T}}\left(P-M_{r}\right)+\left(P-M_{r}\right) A_{r} \\
A_{1}^{\mathrm{T}}\left(P-M_{1}\right)+\left(P-M_{1}\right) A_{1} & -\frac{4 \alpha}{\gamma}\left(P-N_{1}\right) & 0 & 0 \\
\vdots & 0 & \ddots & 0 \\
A_{r}^{\mathrm{T}}\left(P-M_{r}\right)+\left(P-M_{r}\right) A_{r} & 0 & 0 & -\frac{4 \alpha}{\gamma}\left(P-N_{r}\right)
\end{array}\right]<0
$$

and the $2^{r}$ LMIs

$$
\pm \gamma\left(A_{1}^{\mathrm{T}} M_{1}+M_{1} A_{1}\right) \pm \cdots \pm \gamma\left(A_{r}^{\mathrm{T}} M_{r}+M_{r} A_{r}\right) \leqslant Y
$$

Then (75) and (78) are satisfied for all $\left(P, N_{1}, \ldots, N_{r}, M_{1}, \ldots, M_{r}, Y\right) \in \tilde{\mathscr{N}}$.

\section{Remark 12}

As in Corollary 1, (80) can be recast as $2 r n^{2}$ constraints.

Corollary 3

Assume $A+A^{\mathrm{T}}<0$ and $A_{i}+A_{i}^{\mathrm{T}}=0$, let $\beta>0$ satisfy $\beta\left(A+A^{\mathrm{T}}\right)+R<0$, let $\alpha>0$, and define $N_{i}=(\alpha \gamma r)^{-1}\left[\beta\left(A+A^{\mathrm{T}}\right)+R\right]+\beta I$ and $M_{1 i}=M_{2 i}=0, i=1, \ldots, r$. Then $P=\beta I$ satisfies (78).

\section{EXAMPLES}

In this section we use LMI methods to calculate solutions along with optimal scalings for the linear, bounded real, inverse, and Popov bounds, as well as their shifted counterparts. In the case of the inverse and linear bounds, the $\alpha$ scalings must be chosen separately. In Example 1 through Example 3, vertex LMIs were used to obtain the best parameter-independent bound from Proposition 2 (marked LMI), along with the Popov and shifted Popov bounds. 
Example 1

Let

$$
A=\left[\begin{array}{cccc}
-0.0002 & 0.2208 & 0 & 0 \\
-0.2208 & -0.0002 & 0 & 0 \\
0 & 0 & -0.0103 & 1.4322 \\
0 & 0 & -1.4322 & -0.0103
\end{array}\right], \quad A_{1}=\left[\begin{array}{cc}
0_{2 \times 2} & I_{2} \\
-I_{2} & 0_{2 \times 2}
\end{array}\right],
$$

where the uncertainty represents modal coupling. Furthermore, let $R=I_{4}$ and $V=I_{4}$, and let $B_{1}$ and $C_{1}$ be given by

$$
B_{1}=\left[\begin{array}{cc}
I_{2} & 0_{2 \times 2} \\
0_{2 \times 2} & -I_{2}
\end{array}\right], \quad C_{1}=\left[\begin{array}{cc}
0_{2 \times 2} & I_{2} \\
I_{2} & 0_{2 \times 2}
\end{array}\right] .
$$

Each plot in Figure 1 shows the exact worst-case performance along with the LMI bound given by Proposition 2. As can be seen in Figure 1(a), the bounded real bound given by Proposition 4 guarantees stability for $|\delta|<0.0003$. Applying Proposition 8 , the shifted bounded real bound is shown in Figure 1(a) and guarantees stability for all $\delta \in \mathbf{R}$. Next, the Popov bound

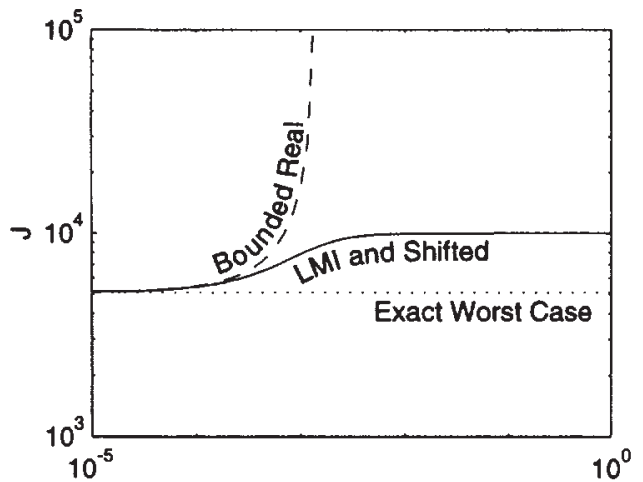

(a)

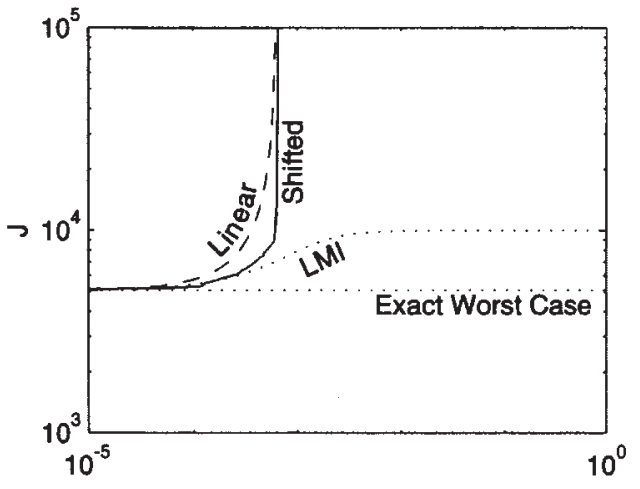

(c)

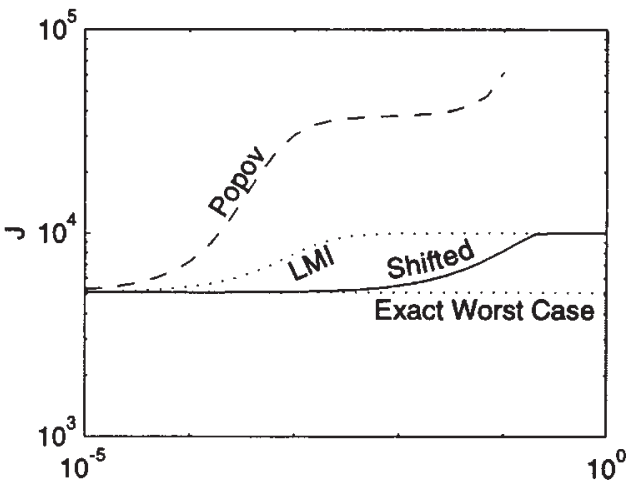

(b)

$\gamma$

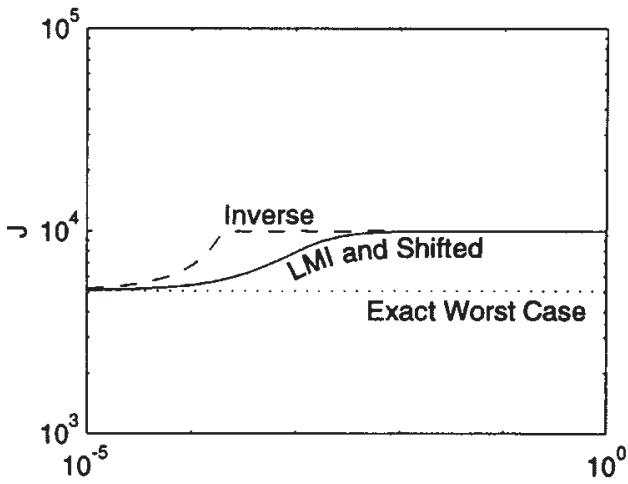

$\gamma$

Figure 1. Comparison of shifted, unshifted, and vertex LMI bounds for Example 1 
in Figure 1(b) guarantees stability for $|\delta|<0.1$, while the shifted Popov bound using Proposition 11 is less conservative than the parameter-independent LMI bound given by Proposition 2. The linear bound in Figure 1(c) guarantees stability for $|\delta|<6 \times 10^{-4}$, while the shifted linear bound is less conservative for $|\delta|<6 \times 10^{-4}$. Finally, the inverse bound in Figure 1(d) guarantees stability for all $\delta \in \mathbf{R}$, while the shifted inverse bound with $M_{1}=0$ coincides with the vertex LMI bound given by Proposition 2. It can be seen that the shifted bounds provide significant improvement over their classical counterparts.

\section{Example 2}

Let

$$
\begin{aligned}
& A=\left[\begin{array}{cc}
-0.005 & 1 \\
-1 & -0.005
\end{array}\right], \quad A_{1}=\left[\begin{array}{cc}
0.001 & 10 \\
-10 & 0.001
\end{array}\right], \\
& R=\left[\begin{array}{cc}
0.25 & 0.12 \\
0.12 & 2.5
\end{array}\right], \quad V=\left[\begin{array}{cc}
0 & 0 \\
0 & 12
\end{array}\right] .
\end{aligned}
$$

Although the uncertainty is nearly skew symmetric, it is destabilizing. Figure 2 shows the exact worst case performance. Now let $B_{1}$ and $C_{1}$ is given by

$$
B_{1}=0.001 I_{2}, \quad C_{1}=\left[\begin{array}{cc}
1 & 10000 \\
-10000 & 1
\end{array}\right] .
$$

With this choice, the bounded real and linear bounds guarantee stability for $|\delta|<4 \times 10^{-4}$, while the shifted linear bound yields an improved robust performance bound for $|\delta|<4 \times 10^{-4}$. The shifted Popov bound, using Proposition 11, guarantees stability for $|\delta|<5$ and is less conservative than the vertex LMI bound. The shifted inverse bound guarantees stability for $|\delta|<5$ and coincides with the vertex LMI bound.

\section{Example 3}

Here we consider several variations of Example 1 with two uncertain parameters. First, consider the non-destabilizing skew-symmetric uncertainties

$$
A_{1}=\left[\begin{array}{cccc}
0 & 0 & 0 & 0 \\
0 & 0 & 0 & 1 \\
0 & 0 & 0 & 0 \\
0 & -1 & 0 & 0
\end{array}\right], \quad A_{2}=\left[\begin{array}{cccc}
0 & 0 & 1 & 0 \\
0 & 0 & 0 & 0 \\
-1 & 0 & 0 & 0 \\
0 & 0 & 0 & 0
\end{array}\right]
$$

and let $B_{0}$ and $C_{0}$ be given by (19), where

$$
B_{1}=\left[\begin{array}{cc}
0 & 0 \\
1 & 0 \\
0 & 0 \\
0 & -1
\end{array}\right], \quad C_{1}=\left[\begin{array}{llll}
0 & 0 & 0 & 1 \\
0 & 1 & 0 & 0
\end{array}\right], \quad B_{2}=\left[\begin{array}{cc}
1 & 0 \\
0 & 0 \\
0 & -1 \\
0 & 0
\end{array}\right], \quad C_{2}=\left[\begin{array}{llll}
0 & 0 & 1 & 0 \\
1 & 0 & 0 & 0
\end{array}\right] .
$$

Figure 3(a) shows the performance bound given by the shifted bounded real bound and the shifted Popov bound, which coincide with the vertex LMI bound given by Proposition 2. 


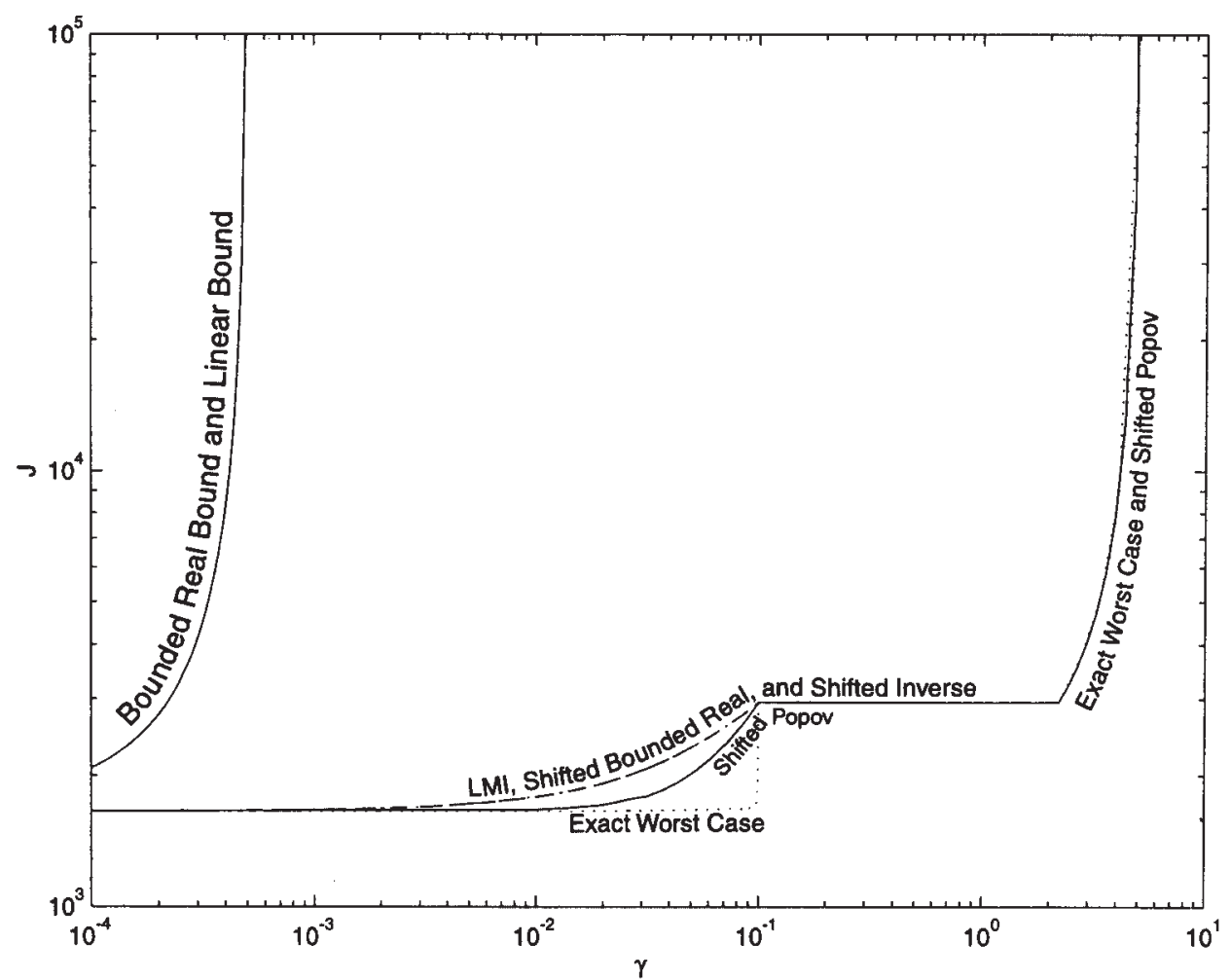

Figure 2. Performance bounds for Example 2 for a destabilizing uncertainty.

Next, consider the symmetric and skew-symmetric uncertainties

$$
A_{1}=\left[\begin{array}{cccc}
0 & 0 & 0 & 0 \\
0 & 0 & 0 & 0.1 \\
0 & 0 & 0 & 0 \\
0 & 0.1 & 0 & 0
\end{array}\right], \quad A_{2}=\left[\begin{array}{cccc}
0 & 0 & 1 & 0 \\
0 & 0 & 0 & 0 \\
-1 & 0 & 0 & 0 \\
0 & 0 & 0 & 0
\end{array}\right],
$$

and let $B_{0}$ and $C_{0}$ be given by (19), where

$$
B_{1}=\left[\begin{array}{cc}
0 & 0 \\
0.1 & 0 \\
0 & 0 \\
0 & 0.1
\end{array}\right]
$$

and $C_{1}, B_{2}$ and $C_{2}$ are given in (82). In this case, the first uncertain parameter is destabilizing. Figure $3(\mathrm{~b})$ shows the performance bound given by the shifted bounded real bound, which coincides with the vertex LMI bound, and the shifted Popov bound, which does slightly better for higher levels of uncertainty. 

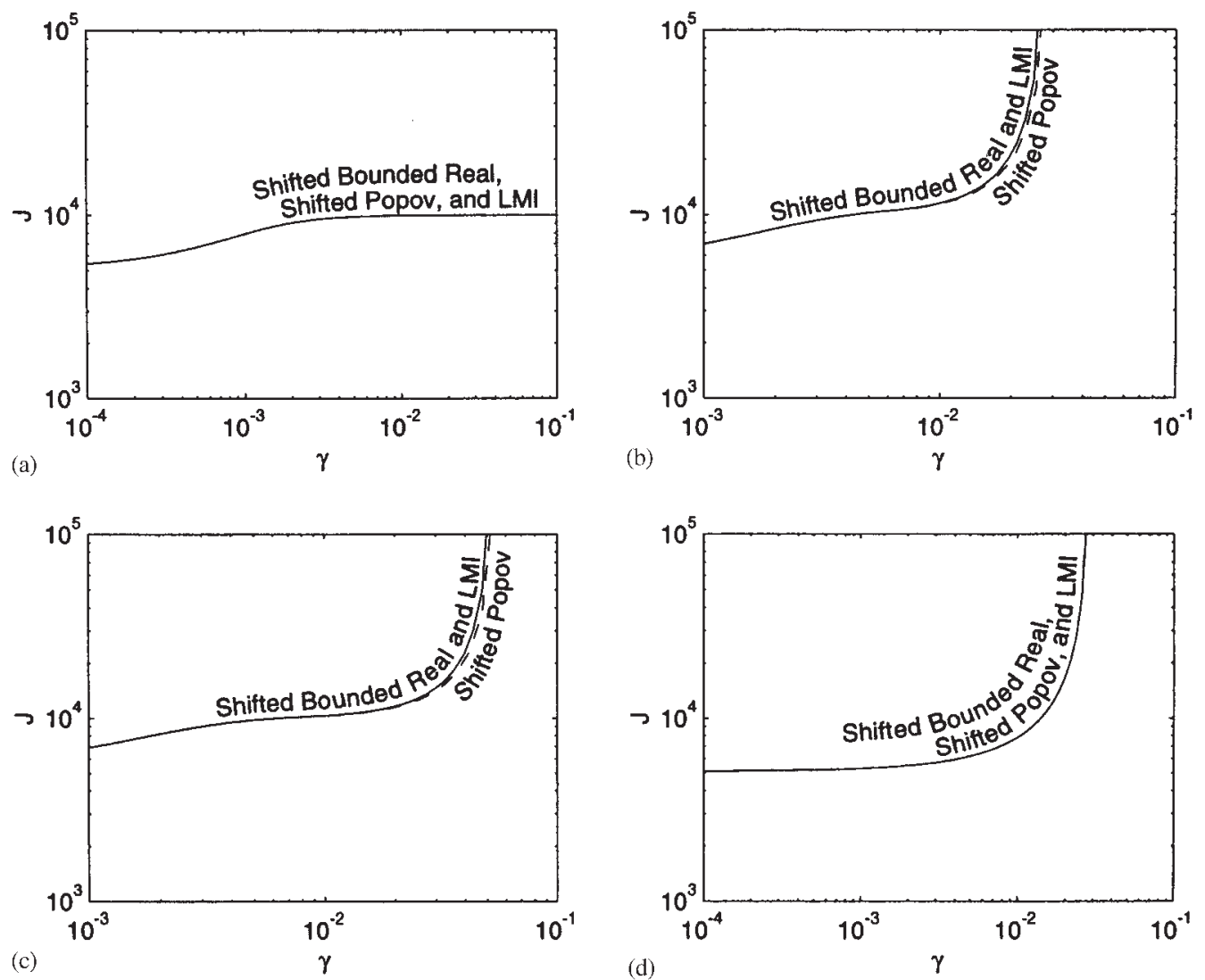

Figure 3. Performance bounds for Example 3 comparing the shifted bounded real, shifted Popov and vertex LMI bounds with two uncertain parameters: (a) two skew-symmetric uncertainties, (b) symmetric and skewsymmetric uncertainty, (c) nilpotent and skew-symmetric uncertainty, (d) two nilpotent uncertainties.

Next, consider the nilpotent and skew-symmetric uncertainties

$$
A_{1}=\left[\begin{array}{cccc}
0 & 0 & 0 & 0 \\
0 & 0 & 0 & 0.1 \\
0 & 0 & 0 & 0 \\
0 & 0 & 0 & 0
\end{array}\right], \quad A_{2}=\left[\begin{array}{cccc}
0 & 0 & 1 & 0 \\
0 & 0 & 0 & 0 \\
-1 & 0 & 0 & 0 \\
0 & 0 & 0 & 0
\end{array}\right],
$$

and let $B_{0}$ and $C_{0}$ be given by (19), where

$$
B_{1}=\left[\begin{array}{c}
0 \\
0.1 \\
0 \\
0
\end{array}\right], \quad C_{1}=\left[\begin{array}{llll}
0 & 0 & 0 & 1
\end{array}\right] \text {, }
$$


and $B_{2}$ and $C_{2}$ are given in (82). In this case, the first uncertain parameter is destabilizing. Figure 3 (c) shows the performance bound given by the shifted bounded real bound, which coincides with the vertex LMI bound, and the shifted Popov bound, which does slightly better for higher levels of uncertainty.

Finally, consider the nilpotent uncertainties

$$
A_{1}=\left[\begin{array}{cccc}
0 & 0 & 0 & 0 \\
0 & 0 & 0 & 0.1 \\
0 & 0 & 0 & 0 \\
0 & 0 & 0 & 0
\end{array}\right], \quad A_{2}=\left[\begin{array}{cccc}
0 & 0 & 0 & 0 \\
0 & 0 & 0 & 0 \\
0.01 & 0 & 0 & 0 \\
0 & 0 & 0 & 0
\end{array}\right]
$$

and let $B_{0}$ and $C_{0}$ be given by (19), where

$$
B_{1}=\left[\begin{array}{c}
0 \\
0.1 \\
0 \\
0
\end{array}\right], \quad C_{1}=\left[\begin{array}{llll}
0 & 0 & 0 & 1
\end{array}\right], \quad B_{2}=\left[\begin{array}{c}
0 \\
0 \\
0.01 \\
0
\end{array}\right], \quad C_{2}=\left[\begin{array}{llll}
1 & 0 & 0 & 0
\end{array}\right] .
$$

In this case, both uncertain parameters are destabilizing. Figure 3(d) shows the performance bound given by the shifted bounded real bound and the shifted Popov bound, which coincide with the vertex LMI bound.

\section{CONCLUSIONS}

In this paper the shifted bounded real bound [18], the shifted linear bound, the shifted inverse bound, and the shifted Popov bound [19] have been considered. These bounds were compared with the bounded real bound, the linear bound, the inverse bound, the Popov bound, and the vertex LMI bound. It was shown that these shifted bounds can be recast as guaranteed cost inequalities described by LMIs. For several examples, it was shown that the shifted bounded real bound and shifted inverse bound are comparable to the best possible parameterindependent bound given by Proposition 2. It has also been shown for several numerical examples that the shifted Popov bound, which is a parameter-dependent bound, may be less conservative than parameter-independent bounds.

Table III lists the various bounds discussed in this paper. The dimensionality of each LMI is compared along with the number of variables required. As can be seen from Table III, there are tradeoffs between the dimension of the constraints and the size of the free variables. The bounded real bound is a more conservative bound and has a lower dimension than the shifted bounded real bound. Similarly, the shifted bounded real bound has a lower dimension than the less conservative Popov and shifted Popov bounds.

\section{ACKNOWLEDGEMENTS}

We wish to thank Siep Weiland for numerous helpful suggestions. This research was supported in part by the Air Force Office of Scientific Research under grant F49620-98-1-0037. 


\section{APPENDIX A}

Proof of Lemma 1

For arbitrary $x \in \mathbf{R}^{n}$, define $f_{x}: \mathscr{R} \rightarrow \mathbf{R}$ by $f_{x}(\delta)=x^{\mathrm{T}}\left[\left(A+\sum_{i=1}^{r} \delta_{i} A_{i}\right)^{\mathrm{T}} P+P\left(A+\sum_{i=1}^{r} \delta_{i} A_{i}\right)+\right.$ $R] x$. Note that $\mathscr{R}$ defined by (24) is the convex hull of the corner points $\mathscr{R}_{\mathrm{H}}$ of a cube in $\mathbf{R}^{r}$. By convexity of $f_{x}, f_{x \mid \mathscr{R}} \leqslant 0$ if and only if $f_{x \mid \mathscr{R}_{\mathrm{H}}} \leqslant 0$. Since $x$ is arbitrary, the result follows.

Proof of Proposition 2

Let $P \in \mathscr{P}$. Thus, from Lemma 1 , an immediate consequence of (28) is

$$
\begin{aligned}
& 0 \leqslant-R-A^{\mathrm{T}} P-P A-\Delta A^{\mathrm{T}} P-P \Delta A \\
& =\Omega_{\mathrm{LMI}}(P)-\Delta A^{\mathrm{T}} P-P \Delta A .
\end{aligned}
$$

Therefore $\left(\Omega_{\mathrm{LMI}}, 0\right)$ is a bounding pair.

Proof of Proposition 3

Since $(\Omega, 0)$ is a bounded pair and $P$ satisfies (6) then

$$
(A+\Delta A)^{\mathrm{T}} P+P(A+\Delta A)+R \leqslant A^{\mathrm{T}} P+P A+\Omega(P)+R \leqslant 0 .
$$

By Lemma $1, P$ satisfies (27). Therefore $P \in \mathscr{P}$, and (28) follows as an immediate consequence.

\section{Proof of Proposition 7}

To prove $(39) \Rightarrow(40)$, suppose (39) is satisfied. Then it follows that, for $i=1, \ldots, r$,

$$
-Y_{i}=-\gamma\left|N_{i}^{\mathrm{T}} C_{i}+C_{i}^{\mathrm{T}} N_{i}\right| \leqslant \gamma\left(N_{i}^{\mathrm{T}} C_{i}+C_{i}^{\mathrm{T}} N_{i}\right) \leqslant \gamma\left|N_{i}^{\mathrm{T}} C_{i}+C_{i}^{\mathrm{T}} N_{i}\right|=Y_{i},
$$

thus (40) is satisfied. Finally, to prove that $(40) \Leftrightarrow(36)$, methods from the proof of Lemma 1 can be used.

Proof of Proposition 11

First, using the technique used in the proof of Lemma 1, it can be shown that if $\bar{P}_{0}$ satisfies (56), then $\bar{P}_{0} \geqslant P_{0}(\Delta A)$ where $P_{0}(\Delta A)$ is given by (48). Similarly, it can be shown that if $Y$ satisfies (55), then $Y$ also satisfies (49). To show that (54) implies (6) with $\Omega(P)$ given by (50), premultiply and postmultiply (54) by $S$ and $S^{\mathrm{T}}$, where $S$ is given by

$$
S=\left[\begin{array}{cc}
I & \left(C_{0}^{\mathrm{T}} H+A_{\mathrm{P}}^{\mathrm{T}} C_{0}^{\mathrm{T}} N+P B_{0}-B_{0} X^{\mathrm{T}}\right) R_{0}^{-1} \\
0 & I
\end{array}\right],
$$

and

$$
R_{0}=\gamma^{-1} H-N C_{0} B_{0}-\left(N C_{0} B_{0}\right)^{\mathrm{T}}>0 .
$$

Now from Theorem 1, it follows that $J(\mathscr{U}) \leqslant \operatorname{tr}\left(P+\bar{P}_{0}\right) V$.

Proof of Corollary 1

The proof uses some of the techniques used in the proof of Lemma 1. 
Proof of Proposition 13

Note that

$$
\begin{aligned}
0 & \leqslant \sum_{i=1}^{r} \alpha^{-1}\left(\delta_{i} A_{i}^{\mathrm{T}}-\alpha I\right)\left(P-N_{i}\right)\left(\delta_{i} A_{i}-\alpha I\right) \\
& =\sum_{i=1}^{r}\left[\alpha\left(P-N_{i}\right)+\alpha^{-1} \delta_{i}^{2} A_{i}^{\mathrm{T}}\left(P-N_{i}\right) A_{i}+\delta_{i}\left(A_{i}^{\mathrm{T}} N_{i}+N_{i} A_{i}\right)\right]-\Delta A^{\mathrm{T}} P-P \Delta A \\
& \leqslant \sum_{i=1}^{r}\left[\alpha\left(P-N_{i}\right)+\alpha^{-1} \gamma^{2} A_{i}^{\mathrm{T}}\left(P-N_{i}\right) A_{i}\right]+Y-\Delta A^{\mathrm{T}} P-P \Delta A \\
& =\Omega(P)-\Delta A^{\mathrm{T}} P-P \Delta A .
\end{aligned}
$$

Proof of Proposition 15

The proof uses some of the techniques used in the proof of Lemma 1.

Proof of Proposition 17

Note that

$$
\begin{aligned}
0 \leqslant & \gamma \alpha \sum_{i=1}^{r}\left[\frac{\delta_{i}}{\gamma}\left(P-N_{i}\right)-\frac{1}{2 \alpha}\left(A_{i}^{\mathrm{T}}\left[P-M_{1 i}\right]+\left[P-M_{2 i}\right] A_{i}\right)\right] \\
& \left(P-N_{i}\right)^{-1}\left[\frac{\delta_{i}}{\gamma}\left(P-N_{i}\right)-\frac{1}{2 \alpha}\left(A_{i}^{\mathrm{T}}\left[P-M_{1 i}\right]+\left[P-M_{2 i}\right] A_{i}\right)\right]^{\mathrm{T}} \\
= & \sum_{i=1}^{r}\left[\frac { \gamma } { 4 \alpha } ( A _ { i } ^ { \mathrm { T } } [ P - M _ { 1 i } ] + [ P - M _ { 2 i } ] A _ { i } ) ( P - N _ { i } ) ^ { - 1 } \left(A_{i}^{\mathrm{T}}\left[P-M_{1 i}\right]\right.\right. \\
& \left.+\left[P-M_{2 i}\right] A_{i}\right)^{\mathrm{T}}+\frac{\alpha}{\gamma} \delta_{i}^{2}\left(P-N_{i}\right)-\frac{\delta_{i}}{2}\left(A_{i}^{\mathrm{T}}\left[2 P-M_{1 i}-M_{2 i}^{\mathrm{T}}\right]\right. \\
& \left.\left.+\left[2 P-M_{2 i}-M_{1 i}^{\mathrm{T}}\right] A_{i}\right)\right] \\
= & \sum_{i=1}^{r}\left[\frac { \gamma } { 4 \alpha } ( A _ { i } ^ { \mathrm { T } } [ P - M _ { 1 i } ] + [ P - M _ { 2 i } ] A _ { i } ) ( P - N _ { i } ) ^ { - 1 } \left(A_{i}^{\mathrm{T}}\left[P-M_{1 i}\right]\right.\right. \\
& \left.\left.+\left[P-M_{2 i}\right]_{i}\right)^{\mathrm{T}}+\frac{\alpha}{\gamma} \delta_{i}^{2}\left(P-N_{i}\right)-\frac{\delta_{i}}{2}\left(A_{i}^{\mathrm{T}}\left[M_{1 i}+M_{2 i}^{\mathrm{T}}\right]+\left[M_{2 i}+M_{1 i}^{\mathrm{T}}\right] A_{i}\right)\right] \\
& -\Delta A^{\mathrm{T}} P-P \Delta A \\
\leqslant & \sum_{i=1}^{r}\left[\frac { \gamma } { 4 \alpha } ( A _ { i } ^ { \mathrm { T } } [ P - M _ { 1 i } ] + [ P - M _ { 2 i } ] A _ { i } ) ( P - N _ { i } ) ^ { - 1 } \left(A_{i}^{\mathrm{T}}\left[P-M_{1 i}\right]\right.\right. \\
& \left.\left.+\left[P-M_{2 i}\right] A_{i}\right)^{\mathrm{T}}+\alpha \gamma\left(P-N_{i}\right)\right]+Y-\Delta A^{\mathrm{T}} P-P \Delta A \\
= & \Omega(P)-\Delta A^{\mathrm{T}} P-P \Delta A .
\end{aligned}
$$


Proof of Proposition 18

Let $P \geqslant 0, \alpha>0, N_{1}, \ldots, N_{r}, M_{1}, \ldots, M_{r} \in \mathbf{S}^{n}$ satisfy (79). To show that (79) is equivalent to (78) with $P \in \mathscr{N}$, premultiply and postmultiply (79) by $S$ and $S^{\mathrm{T}}$, where $S$ is given by

$$
S=\left[\begin{array}{cccc}
I & \frac{\gamma}{4 \alpha}\left[A_{1}^{\mathrm{T}}\left(P-M_{1}\right)+\left(P-M_{1}\right) A_{1}\right]\left(P-N_{1}\right)^{-1} & \cdots & \frac{\gamma}{4 \alpha}\left[A_{r}^{\mathrm{T}}\left(P-M_{r}\right)+\left(P-M_{r}\right) A_{r}\right]\left(P-N_{r}\right)^{-1} \\
0 & I & 0 & 0 \\
\vdots & 0 & \ddots & 0 \\
0 & 0 & 0 & I
\end{array}\right] .
$$

Proof of Corollary 3

Suppose $P=\beta I$. Then (78) becomes

$$
\begin{aligned}
0= & A^{\mathrm{T}} P+P A+\sum_{i=1}^{r} \alpha \gamma\left(P-N_{i}\right)+R \\
& +\frac{\gamma}{4 \alpha} \sum_{i=1}^{r}\left[A_{i}^{\mathrm{T}}\left(P-M_{1 i}\right)+\left(P-M_{2 i}\right) A_{i}\right]\left(P-N_{i}\right)^{-1}\left[A_{i}^{\mathrm{T}}\left(P-M_{1 i}\right)+\left(P-M_{2 i}\right) A_{i}\right]^{\mathrm{T}} \\
= & \beta\left(A^{\mathrm{T}}+A\right)+\sum_{i=1}^{r} \alpha \gamma\left(\beta I-\frac{1}{\alpha \gamma}\left[\frac{\beta}{r}\left(A+A^{\mathrm{T}}\right)+R\right]-\beta I\right)+R \\
= & \beta\left(A^{\mathrm{T}}+A\right)-\frac{\beta}{r} \sum_{i=1}^{r}\left(A+A^{\mathrm{T}}\right) .
\end{aligned}
$$

\section{APPENDIX B: NOMENCLATURE}

$\begin{array}{ll}\mathbf{R}^{d} & d \times 1 \text { real column vectors } \\ \mathbf{R}^{m \times n} & m \times n \text { real matrices } \\ I_{n}, 0_{n}, \mathbf{S}^{n} & n \times n \text { identity matrix, } n \times n \text { zero matrix, } n \times n \text { symmetric matrices } \\ \mathbf{N}^{n}, \mathbf{P}^{n} & n \times n \text { non-negative-definite matrices, } n \times n \text { positive-definite matrices } \\ A \leqslant B, A<B & B-A \text { is non-negative definite, } B-A \text { is positive definite } \\ \operatorname{tr} & \text { trace } \\ |H| & \left(H H^{\mathrm{T}}\right)^{1 / 2}, \text { where } H \in \mathbf{R}^{k_{1} \times k_{2}} \\ \text { vec, } \oplus, \otimes & \text { column stacking operator, Kronecker sum, Kronecker product } \\ {[G, H]} & G H-H G\end{array}$

\section{REFERENCES}

1. Anderson BDO, Vongpanitlerd S. Network Analysis and Synthesis: A Modern Systems Theory Approach. PrenticeHall: Englewood Cliffs, Upper Saddle River, 1973.

2. Noldus E. Design of robust state feedback laws. International Journal of Control 1982; 35:935-944.

3. Petersen IR, Hollot CV. A Riccati equation approach to the stabilization of uncertain systems. Automatica 1986; 22:397-411.

4. Bernstein DS, Haddad WM. Robust stability and performance analysis for state space systems via quadratic Lyapunov bounds. SIAM Journal on Matrix Analysis and Applications 1990; 11:239-271. 
5. Khargonekar PP, Petersen IR, Zhou K. Robust stability of uncertain linear systems: quadratic stability and $H_{\infty}$ control theory. IEEE Transactions on Automatic Control 1990; 35:356-361.

6. Haddad WM, Bernstein DS. Explicit construction of quadratic Lyapunov functions for the small gain, positivity, circle, and Popov theorems and their application to robust stability. Part I: Continuous-time theory. International Journal on Robust Nonlinear Control 1993; 3:313-339.

7. Packard A, Doyle J. Complex structured singular value. Automatica 1993; 29:71-109.

8. Fan M, Tits A, Doyle J. Robustness in the presence of mixed parametric uncertainty and unmodeled dynamics. IEEE Transactions on Automatic Control 1991; 36:25-38.

9. Boyd S, Ghaoui LE, Feron E, Balakrishnan V. Linear Matrix Inequalities in System and Control Theory. SIAM: Philadelphia, 1994.

10. Gahinet P, Apkarian P. A linear matrix inequality approach to $H_{\infty}$ control. International Journal on Robust Nonlinear Control 1994; 4:421-448.

11. Iwasaki T, Skelton RE. All controllers for the general $H_{\infty}$ control problem: LMI existence conditions and statespace formulas. Automatica 1994; 30:1307-1317.

12. Chilali M, Gahinet P. $H_{\infty}$ design with pole placement constraints: an LMI approach. IEEE Transactions on Automatic Control 1996; 41:358-367.

13. Scherer C, Chilali M, Gahinet P. Multiobjective output-feedback control via LMI optimization. IEEE Transactions on Automatic Control 1997; 42:896-910.

14. Gahinet P, Nemirovski A, Laub AJ, Chilali M. LMI Control Toolbox for Use with Matlab. The Mathworks: Natuk, MA, 1995.

15. Megretski A, Rantzer A. System analysis via integral quadratic constraints. IEEE Transactions on Automatic Control 1997; 42:819-830.

16. Anderson BDO. A system theory criterion for positive real matrices. SIAM Journal on Control and Optimization 1967; 5:171-182.

17. Haddad WM, Bernstein DS. Parameter-dependent Lyapunov functions and the Popov criterion in robust analysis and synthesis. IEEE Transactions on Automatic Control 1995; 40:536-543.

18. Tyan F, Bernstein DS. Shifted quadratic guaranteed cost bounds for robust stability and performance. Proceedings of 13th IFAC World Congress, vol. G: Education, Robust Control I, San Francisco, CA, July 1996; 285-290.

19. Kapila V, Haddad WM, Erwin RS, Bernstein DS. Robust controller synthesis via shifted parameter-department quadratic cost bounds. IEEE Transactions on Automatic Control 1998; 43:1003-1007.

20. Haddad WH, Chellaboina V-S, Bernstein DS. An implicit small gain condition and an upper bound for the real structured singular value. Systems \& Control Letters 1997; 29:197-205.

21. Chang SSL, Peng TKC. Adaptive guaranteed cost control of systems with uncertain parameters. IEEE Transactions on Automatic Control 1972; 17:474-483.

22. Jain BN. Guaranteed error estimation in uncertain systems. IEEE Transactions on Automatic Control 1975; 20: 230-232.

23. Bernstein DS. Robust stability and dynamic output-feedback stabilization: deterministic and stochastic perspectives. IEEE Transactions on Automatic Control 1987; 32:1076-1084.

24. Kosmidou OI, Bertrand P. Robust-controller design for systems with large parameter variations. International Journal on Control 1987; 45:927-938.

25. Tyan F, Hall SR, Bernstein DS. A double-commutator guaranteed cost bound for robust stability and performance. Systems \& Control Letters 1995; 25:125-129.

26. Bernstein DS, Haddad WM, Hyland DC, Tyan F. Maximum entropy-type Lyapunov functions for robust stability and performance analysis. Systems \& Control Letters 1993; 21:73-87. 